\title{
The Separate Roles of Geostrophic Vorticity and Deformation in the Midlatitude Occlusion Process
}

\author{
JONATHAN E. MARTIN \\ Department of Atmospheric and Oceanic Sciences, University of Wisconsin-Madison, Madison, Wisconsin
}

(Manuscript received 19 June 1998, in final form 30 October 1998)

\author{
ABSTRACT
}

\begin{abstract}
Separate vector expressions for the rate of change of direction of the potential temperature gradient vector resulting from the geostrophic vorticity and geostrophic deformation, referred to as $\mathbf{Q}_{\mathrm{VR}}$ and $\mathbf{Q}_{\mathrm{DR}}$, respectively, are derived. The evolution of the thermal structure and forcing for quasigeostrophic vertical motion in an occluded cyclone are investigated by examining the distributions of $\mathbf{Q}_{\mathrm{VR}}$ and $\mathbf{Q}_{\mathrm{DR}}$ and their respective convergences.

The dynamics of two common structural transformations observed in the evolution of occluded cyclones are revealed by consideration of these separate forcings. First, the tendency for the sea level pressure minimum to deepen northward and/or westward into the cold air west of the triple point is shown to be controlled by the convergence of $\mathbf{Q}_{\mathrm{VR}}$, which is mathematically equivalent to thermal wind advection of geostrophic vorticity, a well-accepted mechanism for forcing of synoptic-scale vertical motion. Second, the lengthening of the occluded thermal ridge and surface occluded front are forced by the nonfrontogenetic geostrophic deformation, which rotates the cold frontal zone cyclonically while it rotates the warm frontal zone anticyclonically. The net result is a squeezing together of the two frontal zones along the thermal ridge and a lengthening of the occluded thermal ridge. The associated convergence of $\mathbf{Q}_{\mathrm{DR}}$ along the axis of the the thermal ridge also forces vertical motion on a frontal scale. This vertical motion accounts for the clouds and precipitation often observed to extend from the triple point westward to the sea level pressure minimum in the northwest quadrant of occluding cyclones.
\end{abstract}

\section{Introduction}

Extratropical cyclones are often accompanied by frontal baroclinic zones, usually a cold front and a warm front. The thermal evolution of these cyclones represents a central component of their overall structural evolution. This thermal evolution involves changes in the vigor of the individual frontal zones and in their orientation with respect to one another-both of which occur throughout the cyclone life cycle. The dynamical processes that control these two components of the thermal evolution of cyclones are also responsible for producing secondary circulations to which the characteristic cloud and precipitation distribution in cyclones can be accurately ascribed.

Recent work by Keyser et al. (1988) and Keyser et al. (1992), hereafter referred to as K88 and K92, has examined the Lagrangian tendency of the potential temperature gradient vector. K88 derived expressions for the rates of change of both the magnitude and direction of $\boldsymbol{\nabla} \theta$ and called them $\mathbf{F}_{n}$ and $\mathbf{F}_{s}$, respectively. K92 demonstrated that the corresponding quasigeostrophic

Corresponding author address: Dr. Jonathan E. Martin, Department of Atmospheric and Oceanic Sciences, University of Wisconsin-Madison, 1225 W. Dayton St., Madison, WI 53706.

E-mail: jon@meteor.wisc.edu expressions involved the along- and across-isentrope components of the Q-vector (Hoskins et al. 1978) and so could be directly related to forcings for vertical motion through the $\mathrm{Q}-\mathrm{G}$ omega equation.

In a recent study of the dynamics of occluded cyclones, Martin (1999) employed a similar partitioning of the $\mathbf{Q}$-vector into its along- and across-isentrope components ( $\mathbf{Q}_{s}$ and $\mathbf{Q}_{n}$, respectively). He showed that the predominant forcing for upward vertical motions in the occluded sector of midlatitude cyclones was associated with convergence of $\mathbf{Q}_{s}$. It was further shown that the differential rotation of $\boldsymbol{\nabla} \theta$ implied by the convergent field of $\mathbf{Q}_{s}$ was responsible for the production of the characteristic occluded thermal ridge.

In that study it was noted that $\mathbf{Q}_{s}$ was the sum of contributions from both the geostrophic vorticity and deformation (a point first made by K88). These two forcings were not, however, investigated separately. In this paper, we extend our investigation of the rotational (i.e., $\mathbf{Q}_{s}$ ) component of $\mathbf{Q}$ by partitioning it into separate vector expressions representing the rates of change of direction of $\boldsymbol{\nabla} \theta$ produced by the geostrophic vorticity and deformation, respectively. The results of this analysis shed new light on the Q- $\mathrm{G}$ dynamics of the occlusion process by suggesting different, but complementary, roles are played by the geostrophic vorticity and deformation in forcing the thermal evolution, and cloud 
and precipitation distribution, of the postmature phase midlatitude cyclone.

The analysis begins with a brief review of the various forms of the Q-G omega equation in section 2 . We then offer a derivation of the separate vorticity and deformation contributions to $\mathbf{Q}_{s}$ (the rotational component of the $\mathrm{Q}-\mathrm{G}$ vector frontogenesis function) in section 3. Examples of the separation of these two rotational components in the three cyclones investigated by Martin (1999) will also be given there. The evolution of these separate forcings throughout the life cycle of one of these occluding cyclones will be given in section 4 . A discussion of the occlusion process in light of this analysis as well as the development of a dynamic conceptual model of the occlusion process are given in section 5 . Finally, in section 6 conclusions are offered along with suggested future directions.

\section{The quasigeostrophic omega equation}

The traditional form of the $\mathrm{Q}-\mathrm{G}$ omega equation is given by (Holton 1992)

$$
\begin{aligned}
& \left(\sigma \nabla^{2}+f_{o}^{2} \frac{\partial^{2}}{\partial p^{2}}\right) \omega \\
& \quad=f_{o} \frac{\partial}{\partial p}\left[\mathbf{V}_{g} \cdot \nabla\left(\zeta_{g}+f\right)\right]-\nabla^{2}\left[\mathbf{V}_{g} \cdot \nabla\left(\frac{\partial \phi}{\partial p}\right)\right]
\end{aligned}
$$

where $\nabla^{2}=\partial^{2} / \partial x^{2}+\partial^{2} / \partial y^{2}, \zeta_{g}=\left(1 / f_{o}\right) \nabla^{2} \phi$, and $\partial \phi / \partial p$ $=-R T / p$. It is tempting to consider the two terms on the rhs of (1) as representations of separate physical processes; however, a convincing argument against such a practice was made by Trenberth (1978) and Hoskins et al. (1978). Upon carrying out the derivatives on the rhs of (1), Trenberth (1978) found that some cancellation existed between the two terms. By neglecting the socalled "deformation term" (Wiin-Nielsen 1959) he concluded that the rhs of (1) could be approximated by considering the advection of geostrophic absolute vorticity by the column thermal wind, a result similar to that produced by Sutcliffe (1947). The Trenberth (1978) expression of (1) is given by

$$
\begin{aligned}
& \left(\sigma \nabla^{2}+f_{o}^{2} \frac{\partial^{2}}{\partial p^{2}}\right) \omega \\
& =2 f_{o} \frac{\partial \mathbf{V}_{g}}{\partial p} \cdot \nabla\left(\zeta_{g}+f\right) \\
& \quad \mathrm{A} \\
& +2 f_{o}\left[\frac{\partial \mathbf{V}_{g}}{\partial y} \cdot \nabla\left(\frac{\partial U_{g}}{\partial p}\right)-\frac{\partial \mathbf{V}_{g}}{\partial x} \cdot \nabla\left(\frac{\partial V_{g}}{\partial p}\right)\right],
\end{aligned}
$$

which is approximately equal to

$$
\left(\sigma \nabla^{2}+f_{o}^{2} \frac{\partial^{2}}{\partial p^{2}}\right) \omega \approx 2 f_{o} \frac{\partial \mathbf{V}_{g}}{\partial p} \cdot \nabla \zeta_{g}
$$

on an $f$-plane except in frontal-jet streak regions where the neglected deformation term [term B in (2)] is usually large (Wiin-Nielsen 1959). In his examination of the importance of the deformation term throughout the life cycle of a typical midlatitude cyclone, Martin (1998a) has recently offered the more general statement that the deformation term is large in regions where first-order discontinuities in temperature are coincident with regions of nonzero first derivatives in the geostrophic wind field. Such regions are not constrained to be frontal in nature; in fact, he showed that the thermal ridge commonly associated with the occluded quadrant of cyclones is an example of a nonfrontal region in which the deformation term is large in the midtroposphere.

An alternative version of the $\mathrm{Q}-\mathrm{G}$ omega equation on an $f$-plane was given by Hoskins et al. (1978) as

$$
\left(\sigma \nabla^{2}+f_{o}^{2} \frac{\partial^{2}}{\partial p^{2}}\right) \omega=-2 \nabla \cdot \mathbf{Q}
$$

where $\mathbf{Q}$ is given by

$$
\mathbf{Q}=f_{o} \gamma\left[\left(-\frac{\partial \mathbf{V}_{g}}{\partial x} \cdot \boldsymbol{\nabla} \theta\right) \hat{i},\left(-\frac{\partial \mathbf{V}_{g}}{\partial y} \cdot \boldsymbol{\nabla} \theta\right) \hat{j}\right]
$$

with $\gamma=\left(R / f P_{o}\right)\left(P_{o} / P\right)^{c_{v} / c_{p}}$. Thus, the divergence of the $\mathbf{Q}$ vector describes the complete forcing for the $\mathrm{Q}-\mathrm{G}$ omega equation. Another important physical meaning of the $\mathbf{Q}$ vector is that it represents the rate of change of $\boldsymbol{\nabla} \theta$ following the geostrophic flow [i.e., $\mathbf{Q}=$ $f_{o} \gamma\left(d / d t_{g}\right) \boldsymbol{\nabla} \theta$, where $\left.d / d t_{g}=\partial / \partial t+U_{g} \partial / \partial x+V_{g} \partial / \partial y\right]$. As such, $\mathbf{Q}$ describes changes in the magnitude of $\boldsymbol{\nabla} \theta$ as well as changes in the direction of $\boldsymbol{\nabla} \theta$. For this reason, $\mathbf{Q}$ represents the $\mathrm{Q}-\mathrm{G}$ analog of the vector frontogenesis function, F, introduced by K88.

Following a suggestion made by K88, K92 partitioned the $\mathbf{Q}$ vector into along- and across-isentrope components and investigated the vertical motion forcings described by each component in an idealized model simulation. Barnes and Colman (1993) and Kurz (1997) offer recent examples of this partitioning in observed cases. We adopt a natural coordinate system in which $\hat{\mathbf{n}}$ is directed along $\boldsymbol{\nabla} \theta$ and $\hat{\mathbf{s}}$ is $90^{\circ}$ counterclockwise from $\hat{\mathbf{n}}$ (slightly different from that used by K88 and K92). In such a coordinate system the across- and alongisentrope components of $\mathbf{Q}\left(\mathbf{Q}_{n}\right.$ and $\mathbf{Q}_{s}$, respectively) are given by

$$
\mathbf{Q}_{n}=\frac{\mathbf{Q} \cdot \boldsymbol{\nabla} \theta}{|\boldsymbol{\nabla} \theta|}\left[\frac{\boldsymbol{\nabla} \theta}{|\boldsymbol{\nabla} \theta|}\right]=Q_{n} \hat{\mathbf{n}}
$$

and

$$
\mathbf{Q}_{s}=\frac{\mathbf{Q} \cdot(\hat{k} \times \boldsymbol{\nabla} \theta)}{|\boldsymbol{\nabla} \theta|}\left[\frac{(\hat{k} \times \boldsymbol{\nabla} \theta)}{|\boldsymbol{\nabla} \theta|}\right]=Q_{s} \hat{\mathbf{s}}
$$


K92 found that bands of $\mathbf{Q}$ forcing distributed parallel to the baroclinic zones within their idealized cyclone (physically reminiscent of frontal circulations) were entirely accounted for by convergence of the across-isentrope component, $\mathbf{Q}_{n}$. Consistent with this inference, the scalar function $Q_{n}$ is identically equal to the $\mathrm{Q}-\mathrm{G}$ frontogenesis function $\left[F_{g}=\left(d / d t_{g}\right)|\nabla \theta|\right]$. As a result, $Q_{n}$ can be rewritten in terms of the kinematic properties of the geostrophic flow as $Q_{n}=F_{g}=(|\nabla \theta| / 2)\left[E_{g}\right.$ $\cos 2 \beta]$, where $E_{g}$ is the total resultant geostrophic deformation and $\beta$ is the angle between the isentropes and the local axis of dilatation. This formulation reiterates a point implicit in Petterssen (1936); namely, that in purely geostrophic flow the magnitude of the potential temperature gradient vector can be changed only by the geostrophic deformation.

K92 also found that the forcing associated with the along-isentrope component, $\mathbf{Q}_{s}$, was distributed in a dipole on the scale of the synoptic disturbance in their model. They interpreted this fact to mean that convergence of $\mathbf{Q}_{s}$ described the synoptic-scale forcing for Q-G omega. They also showed that the function $Q_{s}$ can be written as $Q_{s}=|\nabla \theta| d \alpha / d t$, where $\alpha$ is the orientation angle of isentropes to the $x$ axis (i.e., a latitude circle). Thus, $Q_{s}$ represents the rotational component of the $\mathrm{Q}-\mathrm{G}$ vector frontogenesis function, with $\boldsymbol{\nabla} \theta$ being rotated counterclockwise (clockwise) for positive (negative) $Q_{s}$. An extension of a derivation presented in K88 shows that $Q_{s}$ can be expressed in terms of invariant kinematic properties of the wind field as

$$
Q_{s}=\frac{|\nabla \theta|}{2}\left(\zeta_{g}+E_{g} \sin 2 \beta\right) .
$$

Thus, the rotational component of the $\mathrm{Q}-\mathrm{G}$ vector frontogenesis function consists of contributions from the geostrophic relative vorticity $\left(\zeta_{g}\right)$ and the resultant geostrophic deformation $\left(E_{g}\right)$. In the next section we will isolate these two contributions in separate vector expressions, each of which contributes to forcing for $\mathrm{Q}-\mathrm{G}$ vertical motion and rotation of $\boldsymbol{\nabla} \theta$.

\section{The vorticity and deformation contributions to $\mathbf{Q}$.}

\section{a. Derivation}

Our partitioning of the rotational component of the Q-G vector frontogenesis function into its vorticity and deformation contributions begins by considering the convergence of $\mathbf{Q}_{s}$. Martin (1999) showed that

$$
-2 \boldsymbol{\nabla} \cdot \mathbf{Q}_{s}=f_{o} \frac{\partial \mathbf{V}_{g}}{\partial p} \cdot \boldsymbol{\nabla} \zeta_{g}+f_{o} \frac{\partial \mathbf{V}_{g}}{\partial p} \cdot \boldsymbol{\nabla}\left(E_{g} \sin 2 \beta\right),
$$

where terms A and B represent the contributions to $\omega$ forcing resulting from the rotation of $\nabla \theta$ made by the geostrophic relative vorticity and the geostrophic deformation, respectively. Term $\mathrm{A}$ is precisely equal to half of the approximate Trenberth forcing for Q-G vertical motion (2a). Martin (1999) noted that this fact accounts for the considerable similarity in distribution that generally exists between regions of Trenberth forcing for upward motion and regions of $\mathbf{Q}_{s}$ convergence in the middle troposphere during the development and early mature stages of a typical midlatitude cyclone.

Hoskins and Pedder (1980) showed that the $f$-plane version of the Trenberth forcing function (2a) could be written in a divergence form similar to that of the $\mathbf{Q}$-vector forcing for $\mathrm{Q}-\mathrm{G}$ omega. That form is given by

$$
2 f_{o} \frac{\partial \mathbf{V}_{g}}{\partial p} \cdot \nabla \zeta_{g}=-2 \boldsymbol{\nabla} \cdot \mathbf{Q}_{\mathrm{TR}},
$$

where $\mathbf{Q}_{\mathrm{TR}}=f_{o} \gamma \zeta_{g}(\hat{k} \times \boldsymbol{\nabla} \theta)$. They did not attribute physical significance to $\mathbf{Q}_{\mathrm{TR}}$ Reference to (6), however, suggests that the vector $\mathbf{Q}_{\mathrm{TR}}$ physically describes twice the rotation of $\boldsymbol{\nabla} \theta$ produced by the geostrophic relative vorticity, a point made by Martin (1999).

Substituting $-\boldsymbol{\nabla} \cdot \mathbf{Q}_{\mathrm{TR}}$ for term A in (6) yields

$$
-2 \boldsymbol{\nabla} \cdot \mathbf{Q}_{s}=-\boldsymbol{\nabla} \cdot \mathbf{Q}_{\mathrm{TR}}+f \frac{\partial \mathbf{V}_{g}}{\partial p} \cdot \boldsymbol{\nabla}\left(E_{g} \sin 2 \beta\right) .
$$

Thus, the forcing for $\omega$ resulting from the rotation of $\nabla \theta$ made by the geostrophic deformation is given by

$$
-2 \boldsymbol{\nabla} \cdot \mathbf{Q}_{s}+\boldsymbol{\nabla} \cdot \mathbf{Q}_{\mathrm{TR}}=f \frac{\partial \mathbf{V}_{g}}{\partial p} \cdot \boldsymbol{\nabla}\left(E_{g} \sin 2 \beta\right)
$$

or

$$
\begin{aligned}
-2 \boldsymbol{\nabla} \cdot\left(\mathbf{Q}_{s}-\frac{1}{2} \mathbf{Q}_{\mathrm{TR}}\right) & \equiv-2 \boldsymbol{\nabla} \cdot \mathbf{Q}_{\mathrm{DR}} \\
& =f \frac{\partial \mathbf{V}_{g}}{\partial p} \cdot \boldsymbol{\nabla}\left(E_{g} \sin 2 \beta\right),
\end{aligned}
$$

where $\mathbf{Q}_{\mathrm{DR}}$ is the vector representing the rate of change of direction of $\boldsymbol{\nabla} \theta$ produced by the geostrophic deformation. It can be shown (see appendixes A and B) that the Cartesian form of $\mathbf{Q}_{\mathrm{DR}}$ is given

$$
\mathbf{Q}_{\mathrm{DR}}=\frac{f_{o} \gamma}{|\boldsymbol{\nabla} \theta|^{2}}\left[E_{1} \frac{\partial \theta}{\partial x} \frac{\partial \theta}{\partial y}+\frac{1}{2} E_{2}\left(\frac{\partial \theta}{\partial y}\right)^{2}-\left(\frac{\partial \theta}{\partial x}\right)^{2}\right](\hat{k} \times \boldsymbol{\nabla} \theta),
$$

where $E_{1}=\left(\partial U_{g} / \partial x-\partial V_{g} / \partial y\right)$ is the geostrophic stretching deformation and $E_{2}=\left(\partial V_{g} / \partial x+\partial U_{g} / \partial y\right)$ is the geostrophic shearing deformation. We shall hereafter refer to the vorticity forcing contribution as $\mathbf{Q}_{\mathrm{VR}}$ noting that $\mathbf{Q}_{\mathrm{VR}}=\frac{1}{2} \mathbf{Q}_{\mathrm{TR}}$.

\section{b. Three examples}

In the study by Martin (1999) three different occluded cyclones were considered and the partitioned $\mathbf{Q}$-vector forcing for each was illustrated at some point after occlusion [see Figs. 14, 15, and 16 of Martin (1999)]. In each case, the vast majority of $\mathrm{Q}-\mathrm{G}$ forcing for ascent 

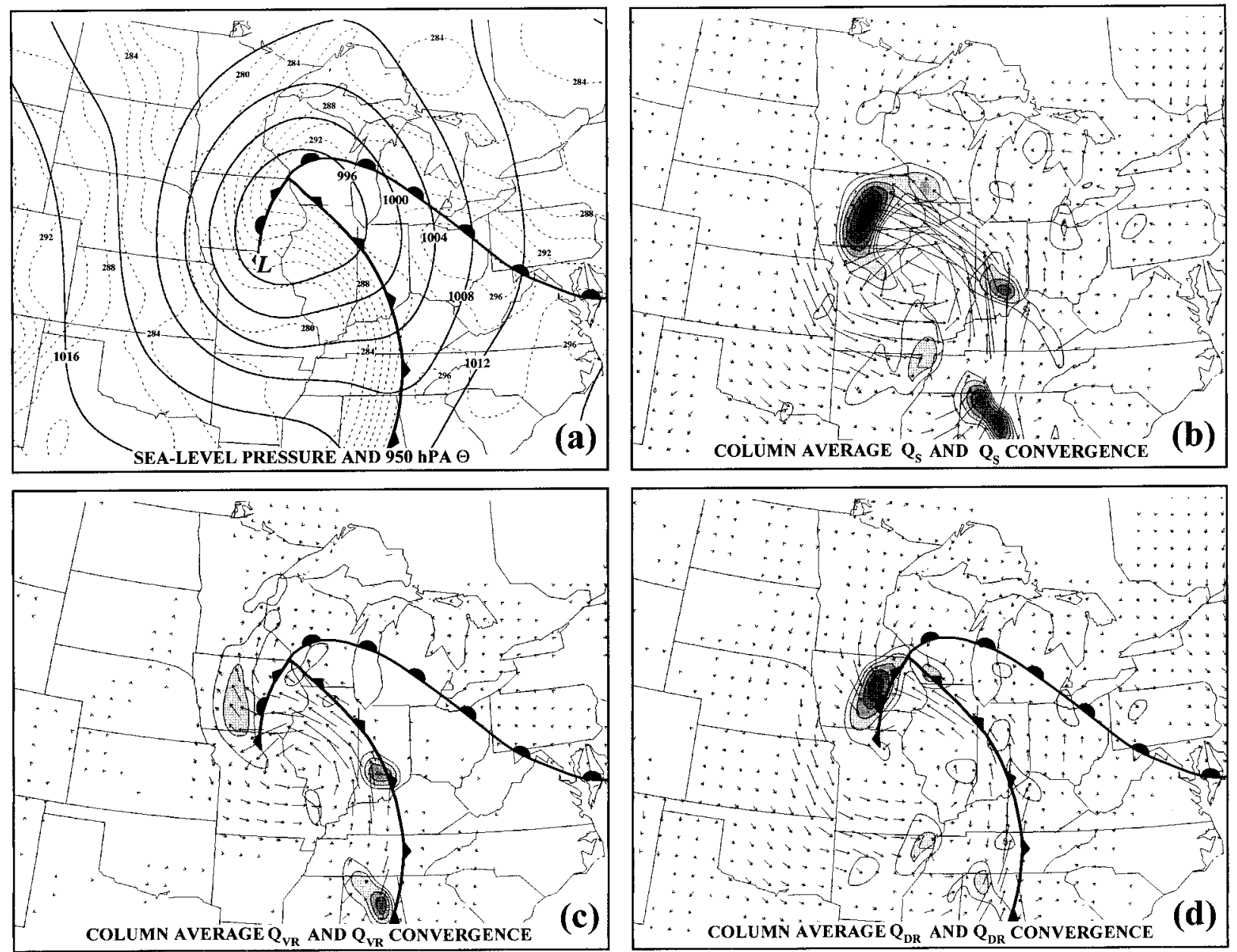

FIG. 1. The 18-h forecast from the UW-NMS valid at 0600 UTC 23 Oct 1996. (a) Solid lines are sea level isobars (labeled in hPa and contoured every $4 \mathrm{hPa}$ ) and dashed lines are 950 -hPa potential temperatures (labeled in $\mathrm{K}$ and contoured every $2 \mathrm{~K}$ ). Conventional frontal analyses indicate model-based positions of the surface fronts. (b) 600-900-hPa column-averaged $\mathbf{Q}$ vectors and $\mathbf{Q}_{\text {s }}$ convergence from an 18-h forecast of the UW-NMS valid at 0600 UTC 23 Oct 1996. The $\mathbf{Q}_{s}$ convergence is contoured and shaded in units of m kg-1 $\mathrm{s}^{-1}$ every $5 \times 10^{-16} \mathrm{~m} \mathrm{~kg}^{-1} \mathrm{~s}^{-1}$ beginning at $5 \times 10^{-16} \mathrm{~m} \mathrm{~kg}^{-1} \mathrm{~s}^{-1}$. (c) The 600-900-hPa column-averaged $\mathbf{Q}_{\mathrm{VR}}$ vectors and $\mathbf{Q}_{\mathrm{VR}}$ convergence from an 18-h forecast of the UW-NMS valid at 0600 UTC 23 Oct 1996. The $\mathbf{Q}_{\mathrm{VR}}$ convergence is contoured and shaded as in Fig. 1b. Surface frontal analysis as in Fig. 1a. (d) The 600-900-hPa column-averaged $\mathbf{Q}_{\mathrm{DR}}$ vectors and $\mathbf{Q}_{\mathrm{DR}}$ convergence from an 18-h forecast of the UWNMS valid at 060023 Oct 1996. The $\mathbf{Q}_{\mathrm{DR}}$ convergence is contoured and shaded as in Fig. 1b. Surface frontal analysis as in Fig. 1a.

in the occluded sector of the cyclone was accounted for by $-2 \boldsymbol{\nabla} \cdot\left(\mathbf{Q}_{s}\right)$. To illustrate the separation between the geostrophic vorticity and deformation contributions to $\mathbf{Q}_{s}$, we show the partitioned $\mathbf{Q}_{s}$ forcing for those same three cyclones in the ensuing figures. It is important to note that the $\mathbf{Q}$-vector forcings are calculated using column-averaged geostrophic wind and $\theta$ in the 500-900hPa layer (except in Fig. 1 where the 600-900 hPa layer is used). The thermal ridge identified by this columnaverage is nearly collocated with the lower-tropospheric thermal ridge used, in part, to determine the position of the surface-occluded front. All of the analyses in this paper employ output from numerical simulations of the selected cyclones performed using the University of Wisconsin Nonhydrostatic Modeling System (UW-NMS). The model description, as well as the specifications for the model runs used to simulate the three cases illustrated here, are described in detail in Martin (1999) and are not repeated here.

At 0600 UTC 23 October 1996 a surface cyclone center was located over the Iowa-Wisconsin-Illinois border. The 950-hPa $\theta$ (Fig. 1a) along with the 950-hPa absolute vorticity (not shown) were used to determine the model forecast frontal positions. The surface occluded front ${ }^{1}$ was located in the $\theta$ ridge extending from

\footnotetext{
${ }^{1}$ It is our opinion that occluded "fronts" do not represent boundaries between different air masses so much as boundaries between different baroclinic zones. Therefore, such boundaries are not "fronts" in the traditional (i.e., air mass) sense of the word. We retain use of the noun "front" as it is the historically accepted term for this feature.
} 

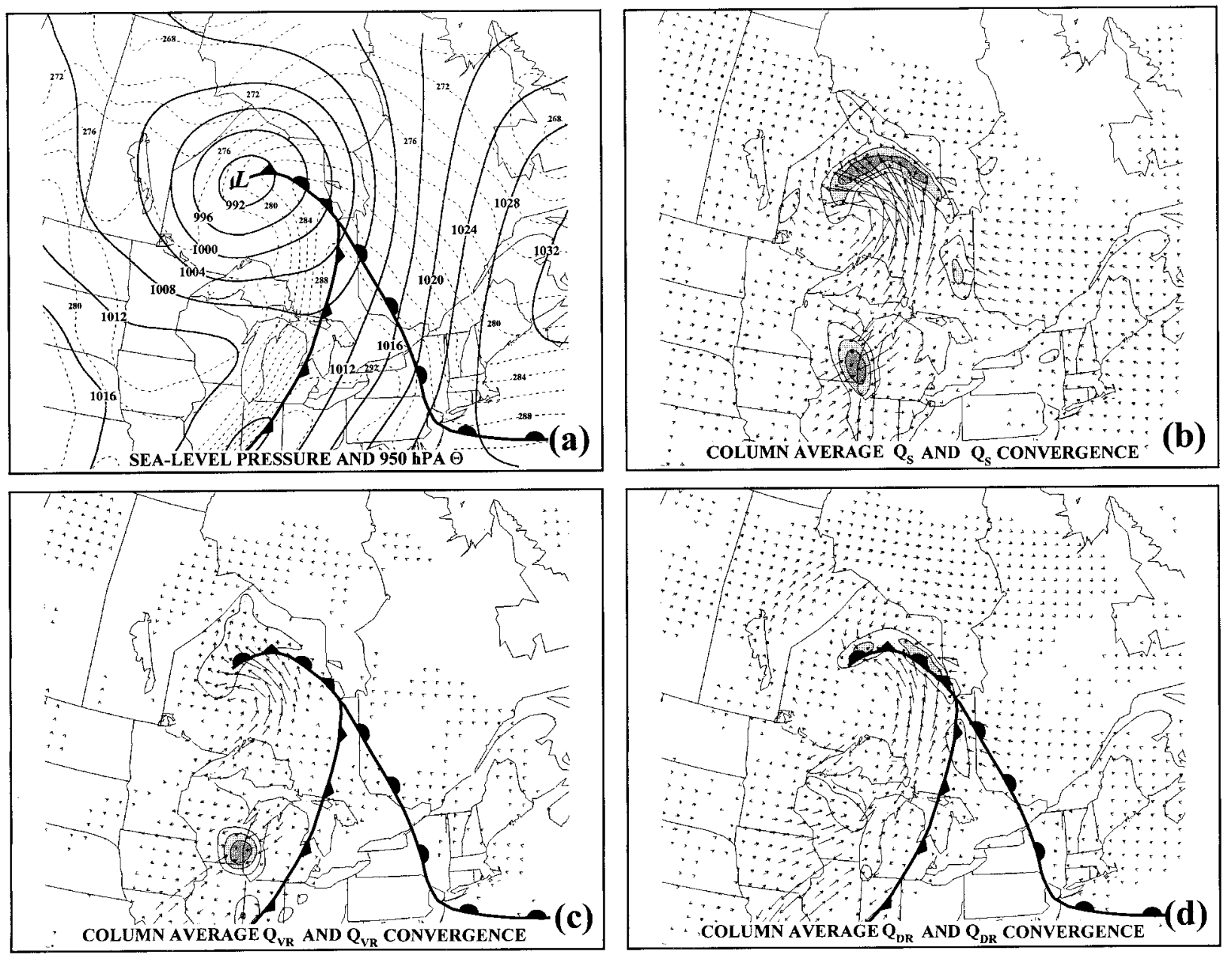

FIG. 2. (a) As for Fig. 1a except from a 12-h forecast by the UW-NMS valid at 1200 UTC 7 Nov 1996. (b) The 500-900-hPa columnaveraged $\mathbf{Q}_{s}$ vectors and $\mathbf{Q}_{s}$ convergence from a 12-h forecast by the UW-NMS valid at 1200 UTC 7 Nov 1996 . The $\mathbf{Q}_{s}$ convergence is contoured and shaded as in Fig. 1b. (c) The 500-900-hPa column-averaged $\mathbf{Q}_{\mathrm{VR}}$ vectors and $\mathbf{Q}_{\mathrm{VR}}$ convergence from a 12 -h forecast by the UW-NMS valid at 1200 UTC 7 Nov 1996. The $\mathbf{Q}_{\mathrm{VR}}$ convergence is contoured and shaded as in Fig. 1b. Surface frontal analysis as in Fig. 2a. (d) The 500-900-hPa column-averaged $\mathbf{Q}_{\mathrm{DR}}$ vectors and $\mathbf{Q}_{\mathrm{DR}}$ convergence from a 12-h forecast by the UW-NMS valid at 1200 UTC 7 Nov 1996. The $\mathbf{Q}_{\mathrm{DR}}$ convergence contoured and shaded as in Fig. 1b. Surface frontal analysis as in Fig. 2a.

extreme northeast Iowa to northern Missouri at this time. The total $\mathbf{Q}_{s}$ forcing is shown in Fig. 1b while the $\mathbf{Q}_{\mathrm{VR}}$ and $\mathbf{Q}_{\mathrm{DR}}$ contributions are shown in Figs. 1c and 1d, respectively. The $\mathbf{Q}_{\mathrm{VR}}$ convergence maximum was located north and west of the surface occluded front (Fig. 1c), while the $\mathbf{Q}_{\mathrm{DR}}$ convergence maximum was nearly coincident with the surface occluded front, ending rather abruptly at the triple point in southwest Wisconsin. Further, the $\mathbf{Q}_{\mathrm{DR}}$ convergence maximum is produced by $\mathbf{Q}_{\mathrm{DR}}$ vectors of roughly the same magnitude but opposing $\hat{\mathbf{S}}$ directions, whereas the $\mathbf{Q}_{\mathrm{VR}}$ convergence maximum (which is of smaller magnitude but larger areal extent) is the result of a diminished magnitude in the vectors, not a change in their direction along the $\hat{\mathbf{s}}$-axis. Importantly, this suggests that the $\mathbf{Q}_{\mathrm{DR}}$ vector field forces opposing rotations of equal magnitude to the component baroclinic zones constituting the sides of the thermal ridge.
The $950-\mathrm{hPa} \theta$ at 1200 UTC 7 November 1996 is shown in Fig. 2a along with the subjectively determined, model-based surface frontal analysis at that time. The total $\mathbf{Q}_{s}$ forcing (Fig. 2b) is once again composed of a vorticity contribution that is located to the northwest of the triple point (Fig. 2c) and a deformation contribution that is collocated with the surface occluded front (Fig. $2 \mathrm{~d})$. It is also interesting to note that the region of forcing for vertical motion associated with the geostrophic vorticity is broad and weak, whereas the forcing for $\omega$ associated with the deformation is much longer than it is wide-suggesting a frontal-type scale. Also, the vorticity forcing for $\omega$ is located over the sea level pressure minimum. As was the case with the previous example, the $\mathbf{Q}_{\mathrm{DR}}$ forcing abruptly ends at the triple point on the southern tip of James Bay.

The most vigorous of the three systems described by Martin (1999) occurred on 1 April 1997. The 950-hPa 
$\theta$ along with the model-based subjectively determined surface fronts for 0600 UTC 1 April 1997 are shown in Fig. 3a. The dashed line at the western end of the occluded front indicates a pressure trough that connects the sea level pressure minimum to a developing secondary cold front off the Carolina coast. The total $\mathbf{Q}_{s}$ forcing at this time is shown in Fig. 3b. The vorticity contribution to this total forcing is shown in Fig. 3c. Consistent with the prior cases, this forcing is located west of the triple point along the western edge of the surface occluded front and is the result of a diminishing magnitude of the $\mathbf{Q}_{\mathrm{VR}}$ vectors, not a systematic change in their direction along the $\hat{\mathbf{s}}$ axis. The vorticity forcing is also, once again, ellipsoidal in shape with major and minor axes of similar lengths. It is also located just to the northwest of the sea level pressure minimum as in the other cases. The $\mathbf{Q}_{\mathrm{DR}}$ vectors themselves are of nearly equal magnitude but different $\hat{\mathbf{s}}$ direction across the surface occluded front resulting in a long and narrow but very intense region of $\mathbf{Q}_{\mathrm{DR}}$ convergence located along the surface occluded front. Also, consistent with the other two cases, the $\mathbf{Q}_{\mathrm{DR}}$ forcing for ascent ends abruptly at the triple point.

The remarkable similarity in the distribution of the $\mathbf{Q}_{\mathrm{VR}}$ and $\mathbf{Q}_{\mathrm{DR}}$ vectors and their respective forcings for vertical motion in these three quite different cyclones testifies to the robust nature of the signal and suggests its relevance for understanding the process of occlusion. Before offering a physical interpretation of these instantaneous distributions, we proceed to examine the $\mathbf{Q}_{\mathrm{VR}}$ and $\mathbf{Q}_{\mathrm{DR}}$ vectors, along with their convergences, throughout the life cycle of the 1 April 1997 cyclone.

\section{Evolution of the separate vorticity and deformation forcings in an occluding cyclone}

In this section we examine the evolution of the components of the $\mathbf{Q}_{s}$ forcing throughout a portion of the life cycle of the 1 April 1997 cyclone. At each time to be shown, the $\mathbf{Q}_{n}$ component of the forcing for $\omega$ in the developing occluded quadrant is much smaller than the $\mathbf{Q}_{s}$ component. As is the case in Figs. 1-3, we show the UW-NMS model-based, subjectively determined surface frontal positions in each of the foregoing figures along with the 500-900-hPa column-averaged $\mathbf{Q}_{s}, \mathbf{Q}_{\mathrm{VR}}$, and $\mathbf{Q}_{\mathrm{DR}}$ vectors at the indicated times.

At 1500 UTC 31 March, a modest sea level pressure minimum was located just offshore of New Jersey and the Delmarva peninsula. The central pressure was 997 $\mathrm{hPa}$ and the lower-tropospheric frontal structure was characteristic of an open wave (Fig. 4a). The $\mathbf{Q}_{s}$ vectors and their convergence were largest just to the northwest of the sea level pressure minimum with significant cyclonic rotation of $\boldsymbol{\nabla} \theta$ along the cold frontal baroclinic zone suggested by the $\mathbf{Q}_{s}$ vectors themselves (Fig. 4b). The vast majority of the $\mathbf{Q}_{s}$ convergence and the cyclonic rotation of $\boldsymbol{\nabla} \theta$ was accounted for by the vorticity contribution (Fig. 4c). Note that the $\mathbf{Q}_{\mathrm{VR}}$ forcing was nearly circular and located north and west of the peak of the warm sector. Very little convergence of $\mathbf{Q}_{D R}$ was evident at this time (Fig. 4d) although the $\mathbf{Q}_{\mathrm{DR}}$ vectors themselves were of opposite $\hat{\mathbf{s}}$ directions along the cold frontal and warm frontal zones, respectively.

In the ensuing $3 \mathrm{~h}$ the lower-tropospheric thermal structure was altered subtly. An incipient thermal ridge had developed, extending northwestward from the peak of the warm sector to Long Island (Fig. 5a). This occluded thermal ridge, in contrast to the thermal ridge located over southern Quebec, connected the surface warm sector to the minimum in sea level pressure. The surface cyclone center (now at $993 \mathrm{hPa}$ ) had moved northeastward to a position south of Long Island and the $\mathbf{Q}_{s}$ forcing for ascent was maximized in its vicinity (Fig. 5b). The $\mathbf{Q}_{\mathrm{VR}}$ contribution to $\mathbf{Q}_{s}$ still provided the majority of the total $\mathbf{Q}_{s}$ forcing and remained to the north and west of the peak of the warm sector, displaced slightly west of the position of the sea level pressure minimum at this time (Fig. 5c). The deformation forcing and $\mathbf{Q}_{\mathrm{DR}}$ vectors are shown in Fig. 5d. Notice the $\mathbf{Q}_{\mathrm{DR}}$ vectors change $\hat{\mathbf{s}}$ direction across the incipient thermal ridge and their convergence is a maximum over its limited length, ending at the peak of the warm sector. The change of $\hat{\mathbf{s}}$ direction of the $\mathbf{Q}_{\mathrm{DR}}$ vectors physically represents the action of the geostrophic deformation in differentially rotating the component baroclinic zones (warm and cold fronts) of this cyclone as the storm develops.

By 0000 UTC 1 April the surface cyclone had intensified to $985 \mathrm{hPa}$ and was located south of Cape Cod, Massachusetts. The 950-hPa $\theta$ demonstrates that by this time the cyclone had occluded (Fig. 6a) as a thermal ridge extended westward from the peak of the warm sector into the sea level pressure minimum. The total $\mathbf{Q}_{s}$ forcing was notable at this time and was maximized from the Delmarva peninsula to well offshore to the southeast of Cape Cod (Fig. 6b). Partitioning of this forcing into its separate vorticity and deformation contributions provides evidence of an emerging pattern. The vorticity forcing was distributed over a large area on the northwestern edge of the new occluded surface front (Fig. 6c). The maximum $\mathbf{Q}_{\mathrm{VR}}$ convergence remained slightly north and west of the sea level pressure minimum, suggesting, in accord with theory, that the eastward progression of the surface cyclone was being retarded by the column stretching associated with the ascent provided by the vorticity component of the $\mathbf{Q}_{s}$ forcing (i.e., thermal wind advection of geostrophic vorticity).

The deformation forcing and the $\mathbf{Q}_{\mathrm{DR}}$ vectors are shown in Fig. 6d. The $\mathbf{Q}_{\mathrm{DR}}$ vectors change $\hat{\mathbf{s}}$ direction across the surface occluded front and thereby provided a narrow, frontal-scale axis of convergence aligned nearly along the surface occluded front, ending at the triple point. The differential rotation of $\nabla \theta$ implied by the distribution of $\mathbf{Q}_{\mathrm{DR}}$ vectors forced a squeezing of the component frontal zones into a thermal ridge connecting 

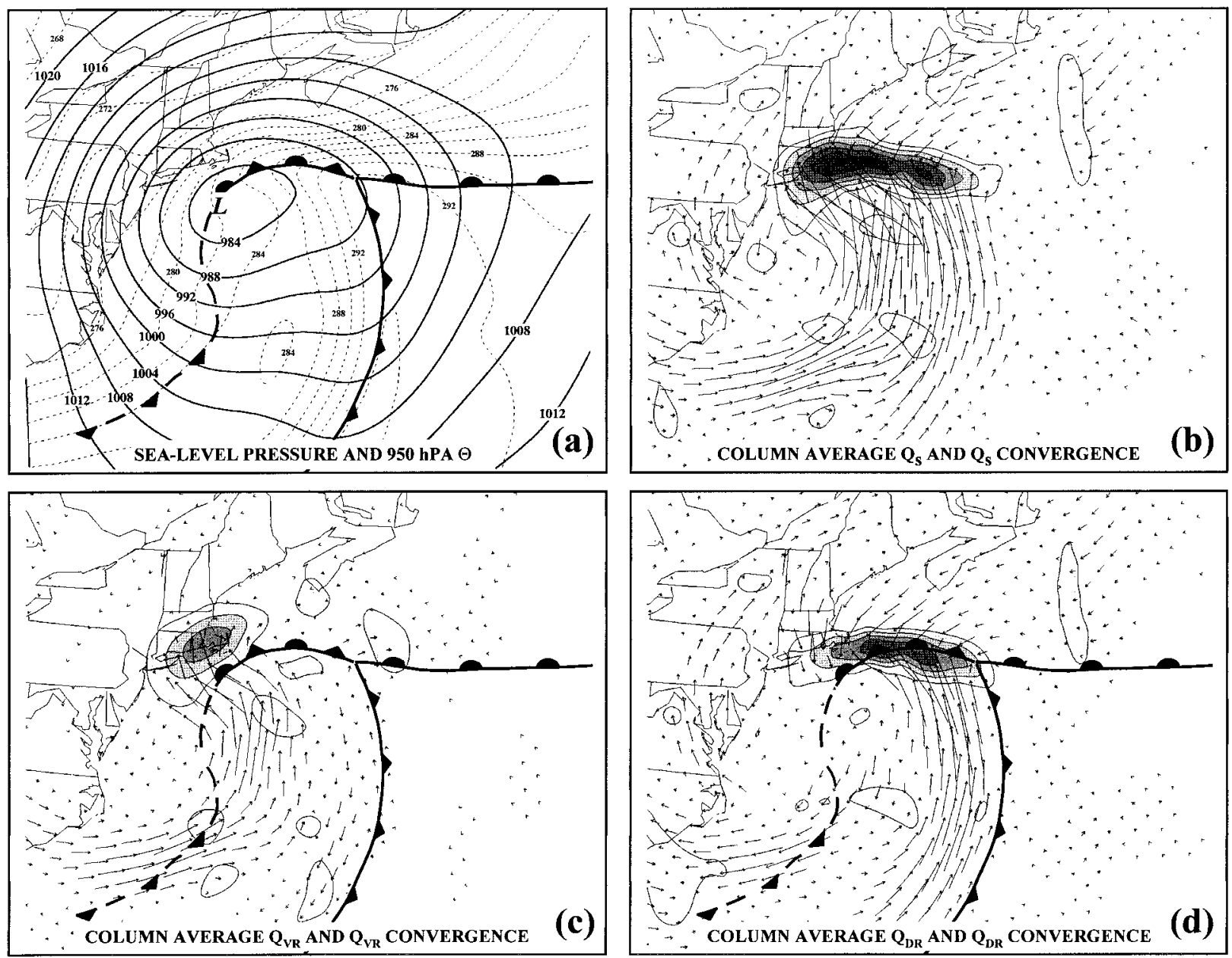

FIG. 3. (a) As for Fig. 2a except from an 18-h forecast by the UW-NMS valid at 0600 UTC 1 Apr 1997. Dashed cold frontal symbol represents the position of the secondary surface cold front. Bold dashed line represents the position of a pressure trough. (b) As for Fig. 2b except from an 18-h forecast by the UW-NMS valid at 0600 UTC 1 Apr 1997. The $\mathbf{Q}_{s}$ convergence is contoured and shaded in units of $\mathrm{m}$ $\mathrm{kg}^{-1} \mathrm{~s}^{-1}$ every $10 \times 10^{-16} \mathrm{~m} \mathrm{~kg}^{-1} \mathrm{~s}^{-1}$ beginning at $5 \times 10^{-16} \mathrm{~m} \mathrm{~kg}^{-1} \mathrm{~s}^{-1}$. (c) As for Fig. 2c except from an 18-h forecast by the UW-NMS valid at 0600 UTC 1 Apr 1997. The $\mathbf{Q}_{\mathrm{VR}}$ convergence is contoured and shaded as in Fig. 3b. Frontal symbols as in Fig. 3a. (d) As for Fig. $2 d$ except from an 18-h forecast by the UW-NMS valid at 0600 UTC 1 Apr 1997. The $\mathbf{Q}_{\mathrm{DR}}$ convergence is contoured and shaded as in Fig. 3b. Frontal symbols as in Fig. 3a.

the triple point to the sea level pressure minimum. Thus, a lengthening and sharpening of the occluded thermal ridge, as well as the vertical motion necessary for the production of the clouds and precipitation that characterized it, were simultaneous results of the geostrophic (nonfrontogenetic) deformation forcing in the vicinity of the thermal ridge.

A similar four-panel diagram corresponding to 0600 UTC 1 April has been shown and described earlier (Fig. 3 ) and fits the exact same pattern as we have described for the other times presented in this section. By 1200 UTC 1 April the surface occluded thermal ridge had lengthened considerably and the sea level pressure minimum (now at $980 \mathrm{hPa}$ ) had correspondingly become further removed from the triple point (Fig. 7a). A secondary cold frontal zone had also become quite obvious in the lower-tropospheric thermal field. The $\mathbf{Q}_{s}$ forcing had become narrow and intense by this time and closely corresponded to the position of the surface occluded front (Fig. 7b). The vorticity contribution to this forcing was, at this time, located well to the west of the triple point and just north and west of the sea level pressure minimum, as had been the case at every stage of the cyclone's evolution.

The $\mathbf{Q}_{D R}$ vectors and their convergence at this time are shown in Fig. 7d. The horizontal scale of the $\mathbf{Q}_{\mathrm{DR}}$ convergence maximum had steadily shrunk during the evolution of this occluded cyclone and was by this time very narrow, aligned along the surface occluded front, ending at the triple point just as it had done at all times subsequent to the original development of the thermal ridge. By this stage in the cyclone life cycle, however, the $\mathbf{Q}_{D R}$ vectors and their convergence constituted the vast majority of the total $\mathbf{Q}_{s}$ forcing. In other words, 

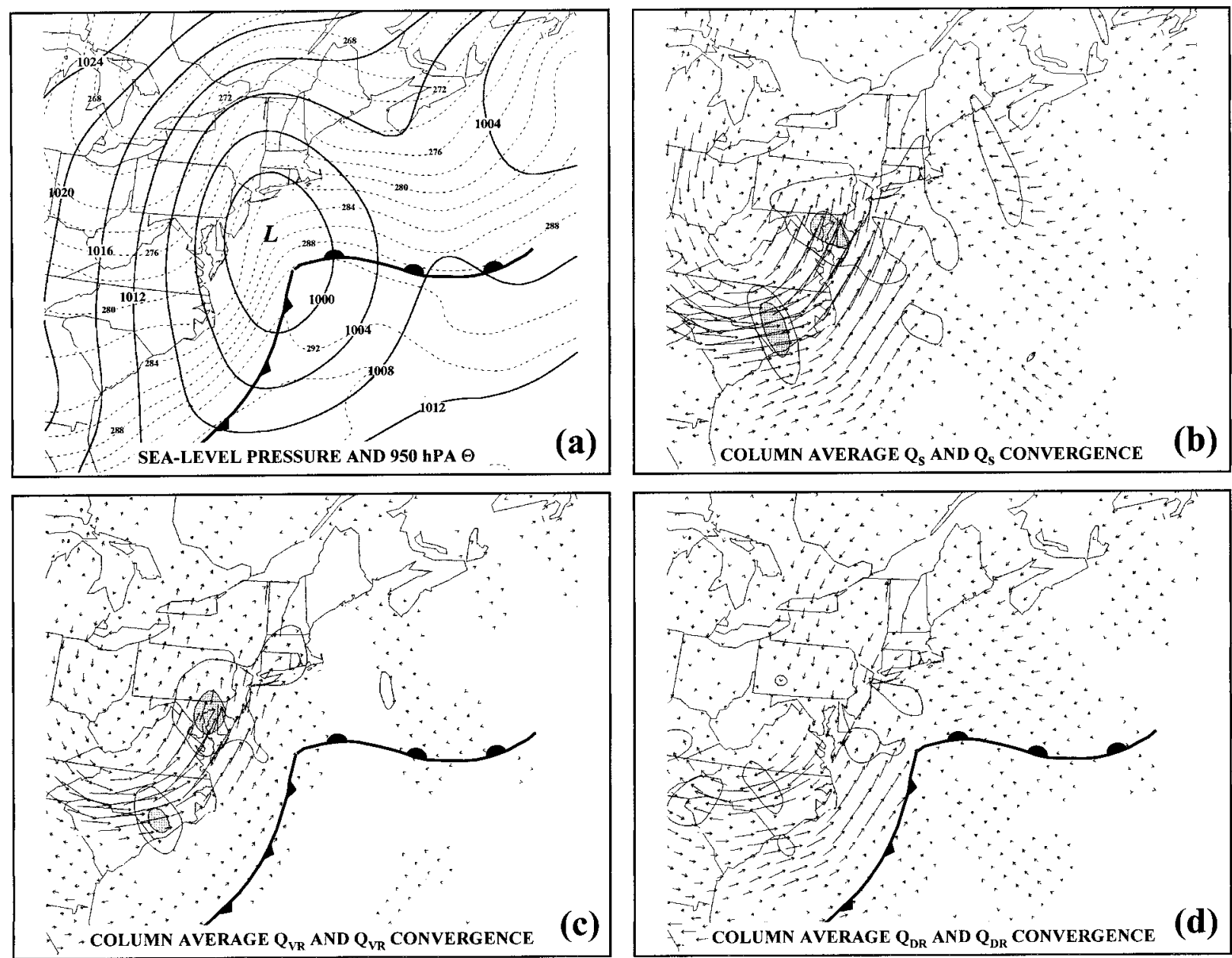

FIG. 4. (a) As for Fig. 3a except from a 3-h forecast by the UW-NMS valid at 1500 UTC 31 Mar 1997. (b) As for Fig. 3b except from a 3-h forecast by the UW-NMS valid at 1500 UTC 31 Mar 1997. (c) As for Fig. 3c except from a 3-h forecast by the UW-NMS valid at 1500 UTC 31 Mar 1997. (d) As for Fig. 3d except from a 3-h forecast by the UW-NMS valid at 1500 UTC 31 Mar 1997.

late in the life cycle, the nonfrontogenetic, geostrophic deformation exerted the largest influence on the total Q-G forcing for ascent in the occluded quadrant of the cyclone. Once again, the $\mathbf{Q}_{\mathrm{DR}}$ vectors changed $\hat{\mathbf{s}}$ direction across the occluded front, implying the importance of the geostrophic deformation in lengthening the thermal ridge by squeezing the component baroclinic zones together along the $\mathbf{Q}_{\mathrm{DR}}$ convergence maximum.

\section{Discussion}

Synoptic experience demonstrates that as a cyclone occludes, two characteristic transformations occur in the lower troposphere: 1) the occluded thermal ridge, which joins the sea level pressure minimum to the triple point, first develops and then lengthens with time, and 2) the sea level pressure minimum "retreats" to the north and west of the triple point, often developing into the cold air. Both the clouds and precipitation that accompany the occluded thermal ridge and the behavior of the sea level pressure minimum are forced by local upward vertical motions. In prior work concerning the $\mathrm{Q}-\mathrm{G}$ forcing for ascent in the occluded sector of cyclones, Martin (1999) showed that the overwhelming majority of the lower- and middle-tropospheric forcing for ascent there is provided by convergence of the along-isentrope component of the $\mathbf{Q}$ vector, $\mathbf{Q}_{s}$. It was also shown that the development of the thermal ridge characteristic of occluded cyclones was a direct consequence of convergence of $\mathbf{Q}_{s}$ since $\mathbf{Q}_{s}$ physically describes the contribution of the geostrophic flow to the rotation of $\nabla \theta$. In this study, the separate vorticity and deformation contributions to the $\mathbf{Q}_{s}$ vector have been isolated in the separate vector expressions, $\mathbf{Q}_{\mathrm{VR}}$ and $\mathbf{Q}_{\mathrm{DR}}$ respectively. The foregoing partitioning of $\mathbf{Q}_{s}$ illustrates that a characteristic distribution of these separate component forcings is involved in the development of an occlusion, suggesting that different, but complementary, roles are played by the geostrophic vorticity and deformation in this process. In fact, it appears that each component of 

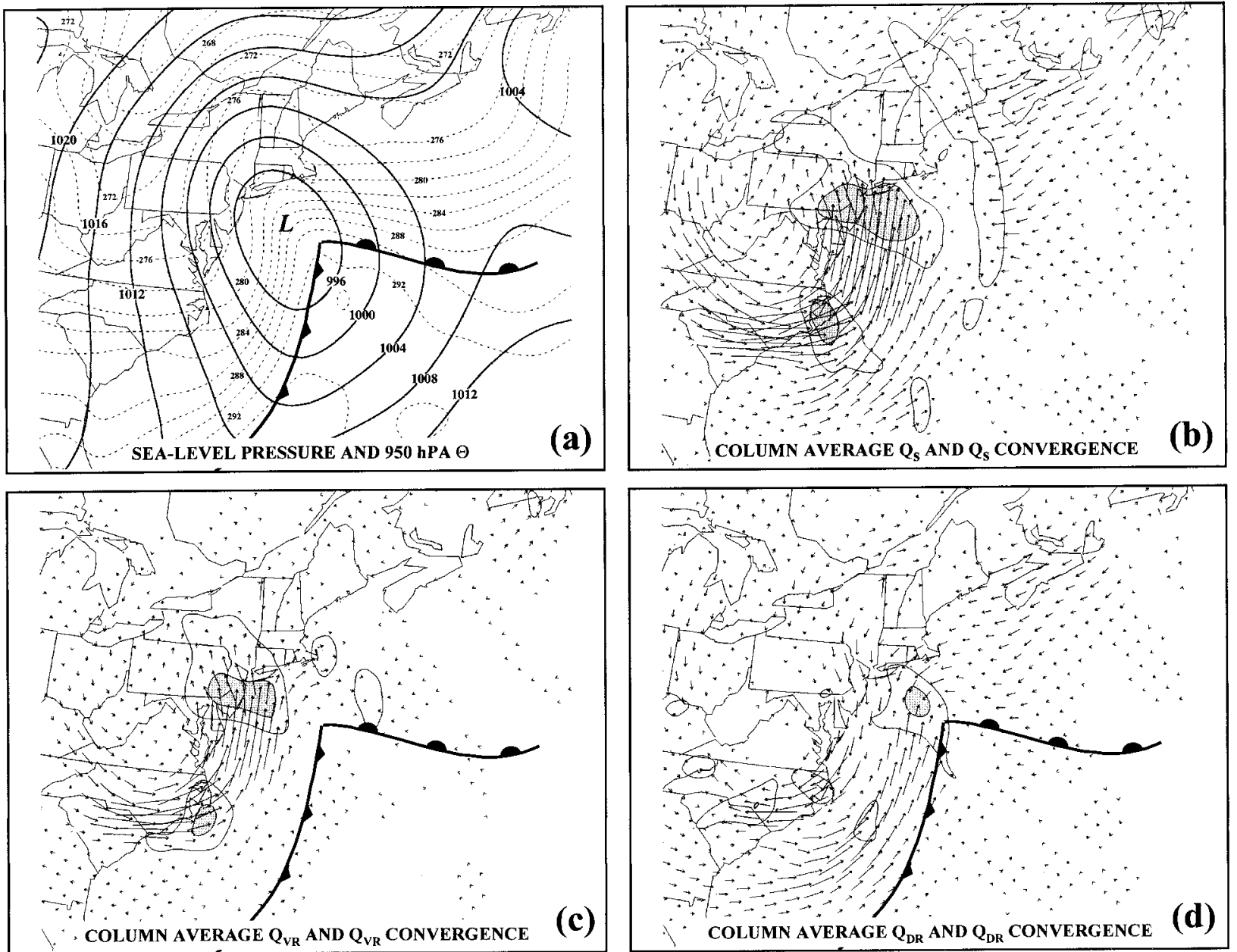

FIG. 5. (a) As for Fig. 3a except from a 6-h forecast by the UW-NMS valid at 1800 UTC 31 Mar 1997. (b) As for Fig. $3 b$ except from a 6-h forecast by the UW-NMS valid at 1800 UTC 31 Mar 1997. (c) As for Fig. 3c except from a 6-h forecast by the UW-NMS valid at 1800 UTC 31 Mar 1997. (d) As for Fig. 3d except from a 6-h forecast by the UW-NMS valid at 1800 UTC 31 Mar 1997.

the forcing plays a central role in one of the two structural transformations mentioned above.

Throughout the evolution depicted in Figs. 4-7, the $\mathbf{Q}_{\mathrm{VR}}$ forcing was located to the north and west of the peak of the warm sector, just northwest of the sea level pressure minimum. The vertical motion that accompanies this component of the baroclinic rotation provides a mechanism for the development of the sea level pressure minimum and its gradual retreat, during the occlusion process, into the cold air north and west of the original warm sector. Thus, as first suggested by Sutcliffe (1947) and later reiterated by Trenberth (1978), the evolution of the sea-level pressure minimum is controlled throughout the cyclone life cycle by the thermal wind advection of geostrophic vorticity.

The sharpening and lengthening of the occluded thermal ridge and the associated development of the occluded front observed in Figs. 4-7, along with the persistence of cloudiness and precipitation in its vicinity (not shown) are simultaneously forced by the nonfrontogenetic geo- strophic deformation according to the distribution of $\mathbf{Q}_{\mathrm{DR}}$ vectors and their convergence. Throughout the evolution described in section 4 , the $\mathbf{Q}_{\mathrm{DR}}$ vectors changed sign ( $\hat{\mathbf{s}}$ direction) across the occluded thermal ridge. Thus, the cold and warm frontal baroclinic zones, which constitute the two sides of the thermal ridge, were rotated with approximately equal magnitude but opposite direction by the geostrophic deformation field. This differential rotation, the result of convergence of the $\mathbf{Q}_{\mathrm{DR}}$ vectors near the thermal ridge, squeezed the warm and cold frontal baroclinic zones together, thereby sharpening and lengthening the thermal ridge and promoting the development of the occluded front. The $\mathbf{Q}_{\mathrm{DR}}$ convergence provided forcing for ascent of the warm sector air that was squeezed between the two baroclinic zones during the process of occlusion. This process is illustrated in schematic form in Fig. 8. It was predominantly this forcing for ascent that accounted for the clouds and precipitation that characterized the occluded quadrant of this cyclone (Martin 1999). 

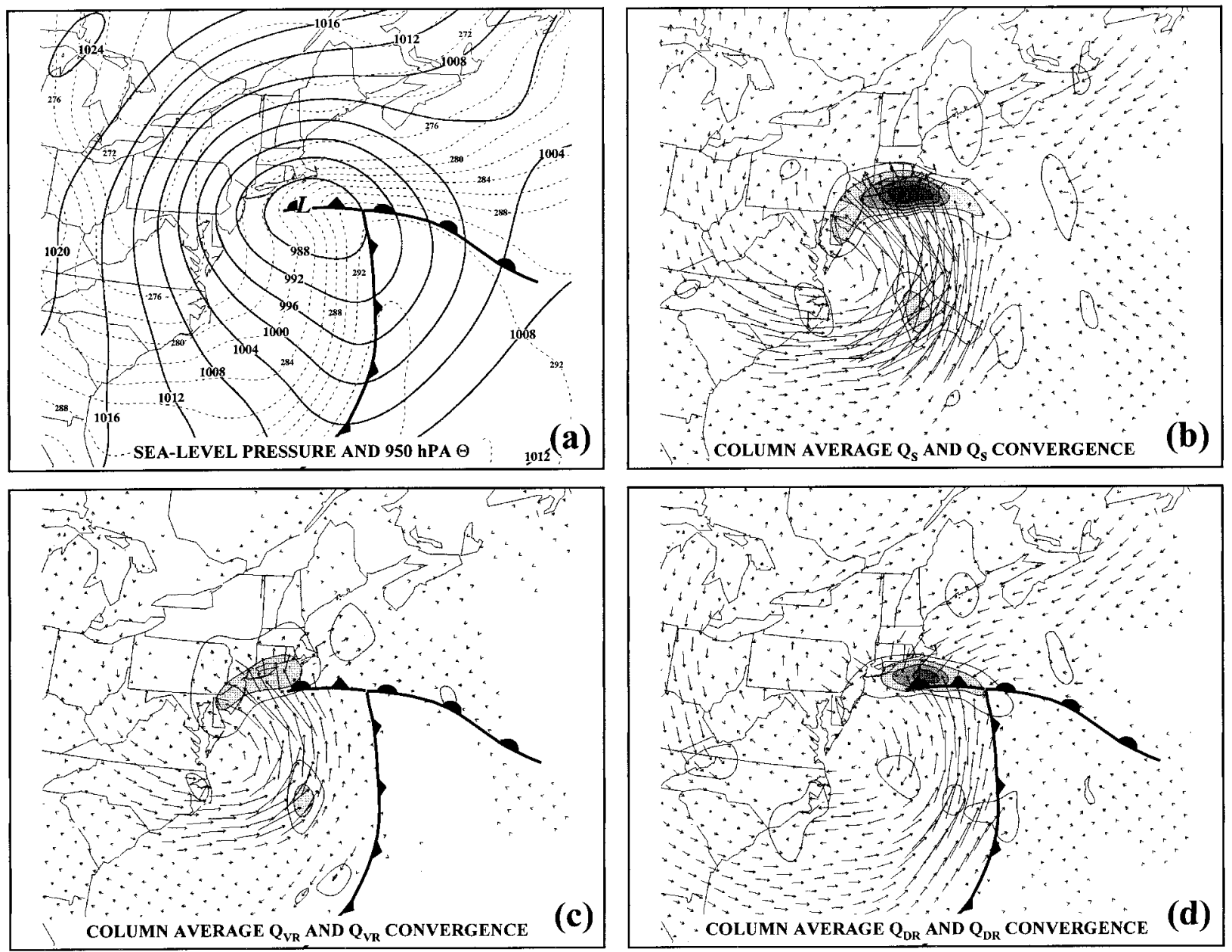

FIG. 6. (a) As for Fig. 3a except from a 12-h forecast by the UW-NMS valid at 0000 UTC 1 Apr 1997. (b) As for Fig. $3 b$ except from a 12-h forecast by the UW-NMS valid at 0000 UTC 1 Apr 1997. (c) As for Fig. 3c except from a 12-h forecast by the UW-NMS valid at 0000 UTC 1 Apr 1997. (d) As for Fig. 3d except from a 12-h forecast by the UW-NMS valid at 0000 UTC 1 Apr 1997.

Another relevant feature of the evolution of the separate $\mathbf{Q}_{\mathrm{VR}}$ and $\mathbf{Q}_{\mathrm{DR}}$ forcings is illustrated in Figs. 4-7. Early in the life cycle (Fig. 4) the developing cyclone was characterized by a broad warm sector and little evidence of a thermal ridge at the peak of the warm sector. At this stage, $\mathbf{Q}_{\mathrm{VR}}$ convergence described nearly all of the total $\mathbf{Q}_{s}$ convergence, suggesting that in the early development stage geostrophic deformation plays a minimal role in baroclinic rotation and its associated vertical motion forcing near the cyclone center. Even at 1800 UTC 31 March (Fig. 5), by which time a more pointed peak to the warm sector had developed, the $\mathbf{Q}_{s}$ forcing was still dominated by the $\mathbf{Q}_{\mathrm{VR}}$ forcing, although a modest contribution by the $\mathbf{Q}_{\mathrm{DR}}$ forcing was precisely collocated with the nascent thermal ridge at that time.

The subsequent evolution of the separate components of $\mathbf{Q}_{s}$ demonstrated the growing importance of $\mathbf{Q}_{\mathrm{DR}}$ convergence to the total $\mathrm{Q}-\mathrm{G}$ vertical motion forcing in the occluded quadrant (Figs. 6, 3, and 7). By 1200 UTC 1 April (Fig. 7) the magnitude of the maximum $\mathbf{Q}_{\mathrm{DR}}$ forc- ing was twice as large as the maximum $\mathbf{Q}_{\mathrm{VR}}$ forcing. The fact that the increasing magnitude of the $\mathbf{Q}_{\mathrm{DR}}$ forcing occurred simultaneously with the development of the thermal ridge is consistent with the recent study of the deformation term in the $\mathrm{Q}-\mathrm{G}$ omega equation by Martin (1998a). He showed that the deformation term can be large, even in the middle troposphere, in regions where second derivatives of $\theta$ are superposed with nonzero first derivatives of the geostrophic wind field. The thermal ridge in the occluded quadrant of the 1 April cyclone possessed both of these characteristics.

Based upon the detailed analysis of the 1 April case presented here, and the analysis of several other cases examined in the course of this research, we propose the following dynamical interpretation of the occlusion process. During the early development stage of a midlatitude cyclone, an upper-level vorticity anomaly encroaches upon a lower-tropospheric baroclinic zone (Fig. 9a). Petterssen and Smebye (1971) described this as a "Type B" development and it is controlled by 

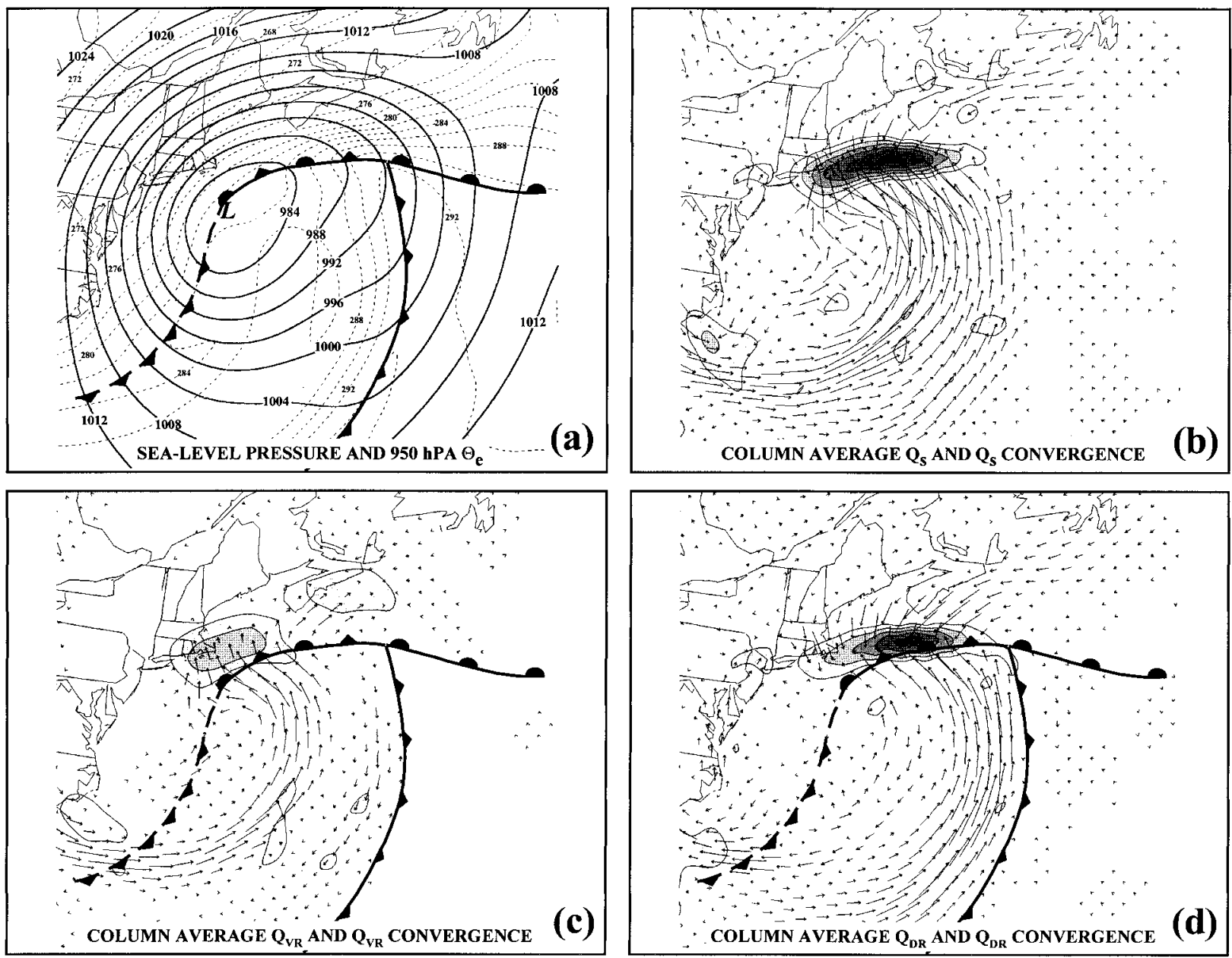

FIG. 7. (a) As for Fig. 3a except from a 24-h forecast by the UW-NMS valid at 1200 UTC 1 Apr 1997. Dashed cold frontal symbol represents the position of the secondary surface cold front. Bold dashed line represents the position of a pressure trough. (b) As for Fig. 3b except from a 24-h forecast by the UW-NMS valid at 1200 UTC 1 Apr 1997. (c) As for Fig. 3c except from a 24-h forecast by the UWNMS valid at 1200 UTC 1 Apr 1997. Frontal symbols as in Fig. 7a. (d) As for Fig. 3d except from a 24-h forecast by the UW-NMS valid at 1200 UTC 1 Apr 1997. Frontal symbols as in Fig. 7a.

thermal wind advection of geostrophic vorticity as shown by Sutcliffe (1947) and Trenberth (1978). The region of cyclonic vorticity advection by the thermal wind is also a region of convergence of $\mathbf{Q}_{\mathrm{VR}}$ with the largest $\mathbf{Q}_{\mathrm{VR}}$ vectors occurring in the maximum vorticity area (Fig. 9b). These $\mathbf{Q}_{\mathrm{VR}}$ vectors represent the contribution of the geostrophic vorticity to rotation of $\boldsymbol{\nabla} \theta$. As such, they differentially rotate the lower-tropospheric baroclinic zone and create a nascent thermal ridge in the region of ascent and height falls downshear of the upper-level vorticity feature (Fig. 9c). Conversely, a thermal trough in the region of descent and height rises is forced upshear of the upper-level vorticity feature.

As soon as the incipient thermal ridge is sufficiently well developed, the deformation forcing $\left(\mathbf{Q}_{\mathrm{DR}}\right)$ grows in magnitude and begins to sharpen and lengthen the occluded thermal ridge by differentially rotating the component baroclinic zones that border it (Fig. 9d). At this stage in the cyclone life cycle, the nonfrontogenetic geostrophic deformation forcing for ascent $\left(-2 \boldsymbol{\nabla} \cdot \mathbf{Q}_{\mathrm{DR}}\right)$ assumes increasing importance as the second derivative of $\theta$ grows in step with the increasing sharpness of the thermal ridge. Sustained ascent associated with the combination of vorticity and deformation forcing ensures that first derivatives of the geostrophic wind field remain collocated with the thermal ridge. The differential rotation of $\boldsymbol{\nabla} \theta$ described by convergence of the $\mathbf{Q}_{\mathrm{DR}}$ vectors results in a squeezing together of the cold and warm frontal baroclinic zones. This process occurs on a smaller scale than the forcing for ascent provided by convergence of $\mathbf{Q}_{\mathrm{VR}}$ and proceeds rapidly to intensify (and lengthen) the occluded thermal ridge, thereby developing the surface occluded front, through a positive feedback that relates the magnitude of the $\mathbf{Q}_{\mathrm{DR}}$ forcing to the magnitude of the second derivative in $\theta$ (Fig. 9e). As the cyclone continues to evolve past the occluded stage, the $\mathbf{Q}_{\mathrm{VR}}$ forcing weakens as vorticity advection lessens. This results in weaker height falls and a weak- 


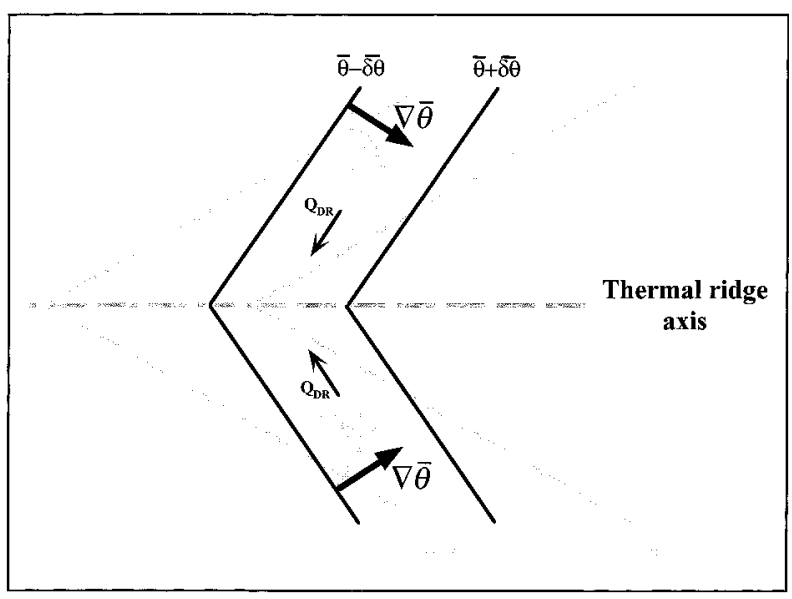

FIG. 8. Schematic illustrating the role of geostrophic deformation in sharpening and lengthening the occluded thermal ridge. Dark (light) solid lines represent column-averaged isentropes at the initial (later) time. The $\mathbf{Q}_{\mathrm{DR}}$ vectors are valid at the initial time. The potential temperature gradient vectors (dark arrows for initial time, light arrows for later time) on either side of the thermal ridge axis are rotated in the direction of $\mathbf{Q}_{\mathrm{DR}}$.

ened geostrophic deformation field in the vicinity of the thermal ridge. This, in turn, leads to weaker $\mathbf{Q}_{\mathrm{DR}}$ forcing and an eventual halt to forcing for ascent in the aging occluded sector.

It was suggested by K92 that a scale separation exists between the forcing for ascent associated with the alongand across-isentrope components of $\mathbf{Q}, \mathbf{Q}_{s}$, and $\mathbf{Q}_{n}$, respectively. The analyses presented here suggest that some of the frontal-scale Q-G forcing for ascent, namely, the $\mathbf{Q}_{\mathrm{DR}}$ forcing, appears to reside within the alongisentrope component of $\mathbf{Q}$. It seems more appropriate, therefore, to suggest instead that an identifiable scale separation exists between the forcings for ascent associated with geostrophic vorticity $\left(\mathbf{Q}_{\mathrm{VR}}\right)$ and geostrophic deformation $\left(\mathbf{Q}_{n}\right.$ and $\left.\mathbf{Q}_{\mathrm{DR}}\right)$. Recognition of this scale separation is implicit in the pioneering works of Petterssen (1936) and Sutcliffe (1947), but is made particularly clear in terms of the foregoing $\mathbf{Q}$-vector partitioning.

Finally, the deformation of $\theta$ contours observed during occlusion suggests that the occlusion process can be viewed as a tropospheric thermal wave-breaking event (Hakim et al. 1996), particularly in light of the definition of wave-breaking given by McIntyre and Palmer (1983) — " the rapid and irreversible deformation of a material contour." In this paper the contributions of the geostrophic vorticity and deformation to this thermal wave-breaking have been isolated through use of the $\mathbf{Q}$ vector, which relates geostrophic wind fields to the conservative (material) variable, $\theta$. The occlusion process also involves a characteristic tropopause potential vorticity (PV) structure, referred to as the "treble clef" by Martin (1998b). The development of this tropopause PV structure results from the breaking of a tropopause PV wave. Since PV is conserved in fric- tionless, adiabatic flow the dynamics of this PV wave breaking could be investigated in a manner similar to that used here by first calculating a vector field, $\mathbf{Q}_{\mathrm{PV}}$, defined as the Lagrangian rate of change of the PV gradient vector following the geostrophic motion:

$$
\mathbf{Q}_{\mathrm{PV}}=\frac{d}{d t_{g}} \boldsymbol{\nabla} P V=\left[-\frac{\partial \mathbf{V}_{g}}{\partial x} \cdot \boldsymbol{\nabla}(P V) \hat{i},-\frac{\partial \mathbf{V}_{g}}{\partial y} \cdot \boldsymbol{\nabla}(P V) \hat{j}\right] .
$$

The development and use of such a diagnostic has recently been discussed by Davies and Rossa (1998). A partition of $\mathbf{Q}_{\mathrm{PV}}$ into its components, $\mathbf{Q}_{\mathrm{PV}_{\mathrm{VR}}}$ and $\mathbf{Q}_{\mathrm{PV}}$ (exactly analogous to the $\mathbf{Q}$-vector partitions, $\mathbf{Q}_{\mathrm{VR}}$ and $\mathbf{Q}_{\mathrm{DR}}$, developed in this paper), would isolate the effects of geostrophic vorticity and deformation, respectively, on the morphological changes in tropopause PV, which are central to the processes of cyclogenesis and occlusion. Such an investigation is currently under way and should provide a new interpretation of, and new insights into, aspects of the dynamics and kinematics of the midlatitude cyclone life cycle.

\section{Conclusions}

In this paper separate vector expressions representing the contributions of the geostrophic vorticity and deformation to the along-isentrope component $\left(\mathbf{Q}_{s}\right)$ of the $\mathbf{Q}$ vector have been isolated. It has been shown that the contribution to the rotation of $\nabla \theta$ made by the geostrophic vorticity can be represented by a vector, $\mathbf{Q}_{\mathrm{VR}}$, the convergence of which is precisely equal to the thermal wind advection of geostrophic vorticity, a fundamental dynamical quantity in midlatitude synoptic development (Sutcliffe 1947; Trenberth 1978). A partitioning of $\mathbf{Q}_{s}$ into its vorticity $\left(\mathbf{Q}_{\mathrm{VR}}\right)$ and deformation $\left(\mathbf{Q}_{\mathrm{DR}}\right)$ contributions in three different cyclones, along with an analysis of their respective evolutions in a single occluded cyclone, were undertaken. The results of this analysis suggest a dynamical explanation of two commonly observed structural transformations associated with occluded midlatitude cyclones.

First, the tendency for the sea level pressure minimum to deepen and migrate northward and westward, into the cold air, after occlusion is controlled by the convergence of $\mathbf{Q}_{\mathrm{VR}}$. This result reaffirms the long-standing synoptic forecasting rule that the behavior of the surface cyclone center is largely controlled by the thermal wind advection of geostrophic (absolute) vorticity (Sutcliffe 1947). Second, convergence of $\mathbf{Q}_{\mathrm{DR}}$ along the thermal ridge axis differentially rotates the cold and warm frontal baroclinic zones that border that axis, rapidly sharpening and lengthening the occluded thermal ridge and leading to the development of the surface occluded front. This convergence forces a narrow, frontal-scale region of ascent that lifts the warm sector air that is squeezed between the cold and warm frontal zones during this process. This lifting is made manifest in the clouds and 

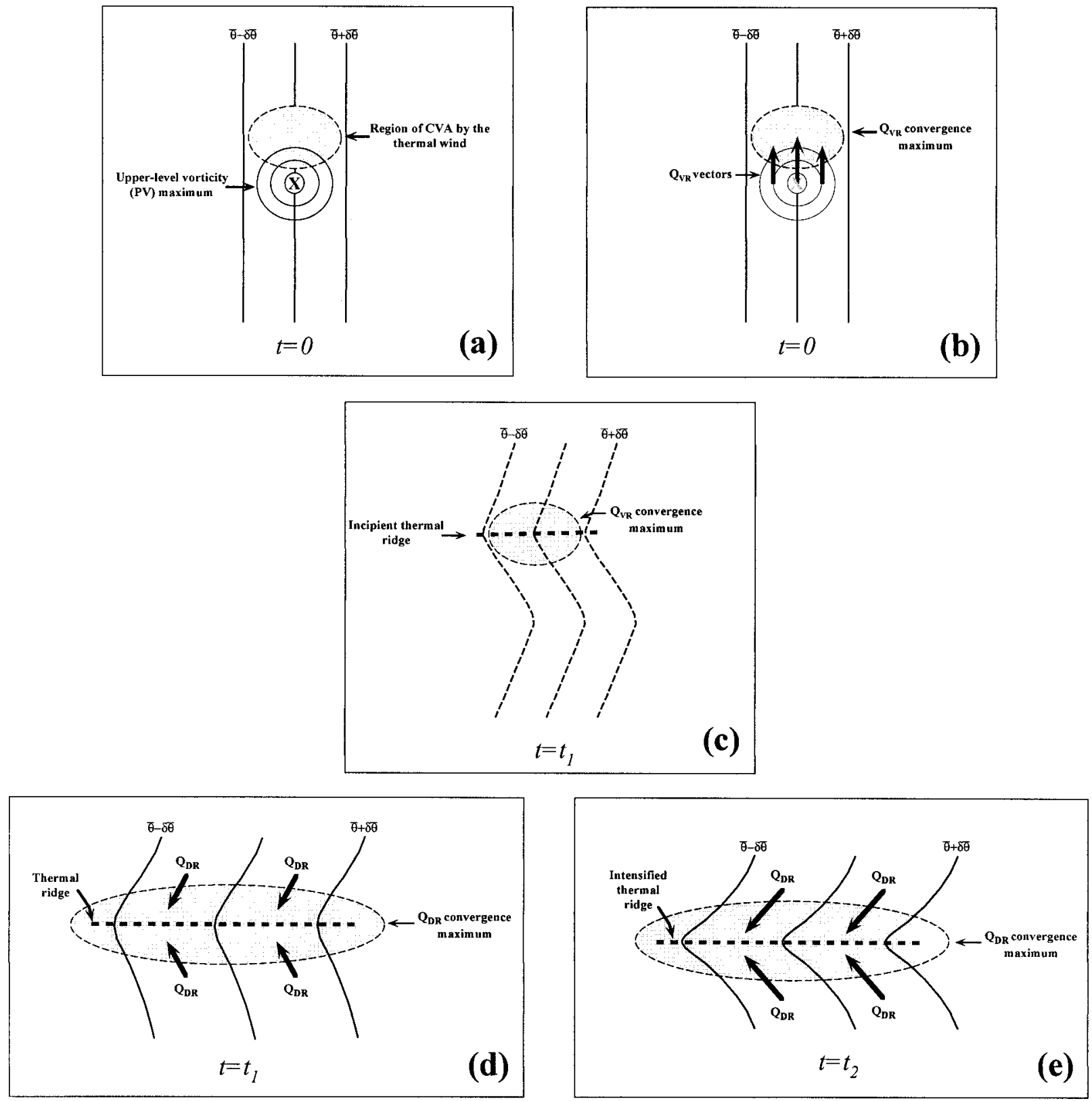

FIG. 9. Schematic illustration of the separate roles played by geostrophic vorticity and deformation in the process of occlusion. (a) Effect of an upper-level vorticity (PV) maximum on development at an initial time, $t=0$. Thick solid lines are column average isentropes, thin solid lines are midtropospheric vorticity (PV) contours with " $\times$ " signifying a cyclonic vorticity maximum. Shading indicates an area of cyclonic vorticity advection (CVA) by the thermal wind. (b) As in Fig. 9a except thick solid arrows are $\mathbf{Q}_{\mathrm{VR}}$ vectors and the shading represents the $\mathbf{Q}_{\mathrm{VR}}$ convergence maximum at $t=0$. (c) Dashed lines are column average isentropes at time $t=t_{1}$ after rotation by the $\mathbf{Q}_{\mathrm{VR}}$ vectors in Fig. 9b. Incipient thermal ridge is located in the region of $\mathbf{Q}_{\mathrm{VR}}$ convergence maximum. (d) Close-up of the thermal ridge in Fig. 9c at $t=$ $t_{1}$. The $\mathbf{Q}_{\mathrm{DR}}$ vectors converge on the thermal ridge axis and force a $\mathbf{Q}_{\mathrm{DR}}$ convergence maximum (shaded) of frontal scale. (e) Thermal ridge at time $t=t_{2}$ intensified through differential rotation implied by $\mathbf{Q}_{\mathrm{DR}}$ vectors in Fig. $9 \mathrm{~d}$. The $\mathbf{Q}_{\mathrm{DR}}$ vectors at $t=t_{2}$ are larger in response to a larger second derivative of $\theta$ in the vicinity of the thermal ridge. Shading represents $\mathbf{Q}_{\mathrm{DR}}$ convergence maximum at $t=t_{2}$.

precipitation that extend along the occluded thermal ridge from the triple point to the sea level pressure minimum. Thus, the distribution of $\mathbf{Q}_{\mathrm{DR}}$ vectors and their convergence in the occluded sector provide both a reaffirmation and dynamical explanation of the traditional synoptic view that the occlusion process involves the squeezing together of the warm and cold frontal zones and a resultant lifting of warm sector air.

The geostrophic vorticity and deformation contributions to baroclinic rotation provide significant, spatially separate forcings for ascent in the occluded quadrant, which, taken together, account for nearly all of the Q-G 
forcing for ascent in the occluded sector of cyclones. The dynamical model of the occlusion process that emerges from this work involves two steps. First, the geostrophic vorticity forces cyclonic rotation of the cold frontal baroclinic zone, production of a nascent thermal ridge, and ascent that initially deepens the surface cyclone. Once the thermal ridge exists, the nonfrontogenetic geostrophic deformation, whose magnitude is dependent on the magnitude of the second derivative of $\theta$, attains greater significance. The increased deformation forcing squeezes the cold and warm frontal baroclinic zones together, sharpening and lengthening the thermal ridge, leading to the development of the characteristic occluded thermal structure. This process is accompanied by forcing for ascent (convergence of $\mathbf{Q}_{\mathrm{DR}}$ ) that occurs on a frontal scale but is a dynamical consequence of the baroclinic rotation forced by the geostrophic deformation.

The results presented here introduce a refinement to an emerging dynamical interpretation of the occlusion process, a process traditionally considered to signal the commencement of cyclone decay. We are currently employing a similarly $\mathbf{Q}$-vector partition to examine the thermal devolution of decaying cyclones within the context of a broader dynamical investigation of cyclolysis.

Acknowledgments. The author wishes to thank Drs. Daniel Keyser, Juan Carlos Jusem, Frederick Sanders, and two anonymous reviewers for numerous thoughtprovoking comments that helped to improve the manuscript. This work was funded by the National Science Foundation under Grant ATM-9505849. It is dedicated to the memory of Mr. Du'o'ng Nhu Lâm, father-in-law of the author.

\section{APPENDIX A}

\section{Derivation of the Cartesian Expression for $Q_{D R}$}

Recall that $\mathbf{Q}_{\mathrm{DR}}=\mathbf{Q}_{s}-\frac{1}{2} \mathbf{Q}_{\mathrm{TR}}$. Therefore,

$\mathbf{Q}_{\mathrm{DR}}=\left[\frac{\mathbf{Q} \cdot(\hat{k} \times \boldsymbol{\nabla} \theta)}{|\boldsymbol{\nabla} \theta|}\left[\frac{(\hat{k} \times \boldsymbol{\nabla} \theta)}{|\boldsymbol{\nabla} \theta|}\right]-\frac{1}{2}\left(f_{o} \gamma \zeta_{g}\right)(\hat{k} \times \boldsymbol{\nabla} \theta)\right]$

or, equivalently,

$\mathbf{Q}_{\mathrm{DR}}=\left[\mathbf{Q} \cdot(\hat{k} \times \boldsymbol{\nabla} \theta)-\frac{1}{2}\left(f_{o} \gamma \zeta_{g}\right)|\boldsymbol{\nabla} \theta|^{2}\right] \frac{(\hat{k} \times \boldsymbol{\nabla} \theta)}{|\boldsymbol{\nabla} \theta|^{2}}$,

where $\mathbf{Q}=f_{o} \gamma\left[\left(-\left(\partial \mathbf{V}_{g} / \partial x\right) \cdot \boldsymbol{\nabla} \theta\right) \hat{i},\left(-\left(\partial \mathbf{V}_{g} / \partial y\right) \cdot \boldsymbol{\nabla} \theta\right) \hat{j}\right]$ and $\hat{k} \times \boldsymbol{\nabla} \theta=(-(\partial \theta / \partial y) \hat{i}$, $(\partial \theta / \partial x) \hat{j})$. Thus,

$$
\begin{aligned}
\mathbf{Q}_{\mathrm{DR}}=\left\{f _ { o } \gamma \left[\left(-\frac{\partial U_{g}}{\partial x} \frac{\partial \theta}{\partial x}-\frac{\partial V_{g}}{\partial x} \frac{\partial \theta}{\partial y}\right)\left(-\frac{\partial \theta}{\partial y}\right)\right.\right. \\
\left.+\left(-\frac{\partial U_{g}}{\partial y} \frac{\partial \theta}{\partial x}-\frac{\partial V_{g}}{\partial y} \frac{\partial \theta}{\partial y}\right)\left(\frac{\partial \theta}{\partial x}\right)\right] \\
\left.-\frac{1}{2} f_{o} \gamma\left(\frac{\partial V_{g}}{\partial x}-\frac{\partial U_{g}}{\partial y}\right)\left[\left(\frac{\partial \theta}{\partial x}\right)^{2}+\left(\frac{\partial \theta}{\partial y}\right)^{2}\right]\right\} \frac{(\hat{k} \times \boldsymbol{\nabla} \theta)}{|\nabla \theta|^{2}}
\end{aligned}
$$

Carrying out all the multiplications and simplifying the resulting expression leads to

$$
\begin{aligned}
\mathbf{Q}_{\mathrm{DR}}=f_{o} \gamma & {\left[\left(\frac{\partial U_{g}}{\partial x}-\frac{\partial V_{g}}{\partial y}\right) \frac{\partial \theta}{\partial x} \frac{\partial \theta}{\partial y}+\frac{1}{2} \frac{\partial V_{g}}{\partial x}\left(\frac{\partial \theta}{\partial y}\right)^{2}\right.} \\
& -\frac{1}{2} \frac{\partial U_{g}}{\partial y}\left(\frac{\partial \theta}{\partial x}\right)^{2}-\frac{1}{2} \frac{\partial V_{g}}{\partial x}\left(\frac{\partial \theta}{\partial x}\right)^{2} \\
& \left.+\frac{1}{2} \frac{\partial U_{g}}{\partial y}\left(\frac{\partial \theta}{\partial y}\right)^{2}\right] \frac{(\hat{k} \times \boldsymbol{\nabla} \theta)}{|\nabla \theta|^{2}} .
\end{aligned}
$$

Substituting the expressions for the stretching and shearing deformations, $E_{1}$ and $E_{2}$, respectively, where $E_{1}=$ $\left(\partial U_{g} / \partial x-\partial V_{g} / \partial y\right)$ and $E_{2}=\left(\partial V_{g} / \partial x+\partial U_{g} / \partial y\right)$ yields

$$
\begin{aligned}
\mathbf{Q}_{\mathrm{DR}}= & f_{o} \gamma\left[E_{1} \frac{\partial \theta}{\partial x} \frac{\partial \theta}{\partial y}+\frac{1}{2} E_{2}\left(\frac{\partial \theta}{\partial y}\right)^{2}-\frac{1}{2} E_{2}\left(\frac{\partial \theta}{\partial x}\right)^{2}\right] \\
& \times \frac{(\hat{k} \times \boldsymbol{\nabla} \theta)}{|\boldsymbol{\nabla} \theta|^{2}}
\end{aligned}
$$

or, finally,

$$
\mathbf{Q}_{\mathrm{DR}}=\frac{f_{o} \gamma}{|\boldsymbol{\nabla} \theta|^{2}}\left\{E_{1} \frac{\partial \theta}{\partial x} \frac{\partial \theta}{\partial y}+\frac{1}{2} E_{2}\left[\left(\frac{\partial \theta}{\partial y}\right)^{2}-\left(\frac{\partial \theta}{\partial x}\right)^{2}\right]\right\} \hat{k} \times \boldsymbol{\nabla} \theta .
$$

\section{APPENDIX B}

\section{An Alternative Derivation of $\mathbf{Q}_{V R}$ and $\mathbf{Q}_{\Delta R}$}

A similar partition of $\mathbf{Q}_{s}$ into its deformation and vorticity components can be obtained following the description given in Davies-Jones (1991). Beginning with

$$
\mathbf{Q}=\frac{1}{2}(\mathbf{Q}+\mathbf{D})+\frac{1}{2}(\mathbf{Q}-\mathbf{D})
$$

where $\mathbf{Q}=f_{o} \gamma\left[\left(-\left(\partial \mathbf{V}_{g} / \partial x\right) \cdot \boldsymbol{\nabla} \theta\right),\left(-\left(\partial \mathbf{V}_{g} / \partial y\right) \cdot \boldsymbol{\nabla} \theta\right)\right]$ and $\mathbf{D}=f_{o} \gamma\left[\left(-\nabla U_{g} \cdot \nabla \theta\right),\left(-\nabla V_{g} \cdot \nabla \theta\right)\right]$. The convergence of $\mathbf{D}$ physically represents the $\mathrm{Q}-\mathrm{G}$ forcing for ascent from the deformation term neglected in the approximate Trenberth (1978) forcing for omega (i.e., $\mathbf{D}=\mathbf{Q}-\mathbf{Q}_{\mathrm{TR}}$ so that $-2 \boldsymbol{\nabla} \cdot \mathbf{D}=-2 \boldsymbol{\nabla} \cdot \mathbf{Q}+2 \boldsymbol{\nabla} \cdot \mathbf{Q}_{\mathrm{TR}}$.

Upon expansion of (B1), the components of the $\mathbf{Q}$ vector become 


$$
\begin{aligned}
\mathbf{Q}_{x}=f_{o} \gamma[ & -\frac{\partial U_{g}}{\partial x} \frac{\partial \theta}{\partial x}-\frac{1}{2}\left(\frac{\partial V_{g}}{\partial x}+\frac{\partial U_{g}}{\partial y}\right) \frac{\partial \theta}{\partial y} \\
& \left.-\frac{1}{2}\left(\frac{\partial V_{g}}{\partial x}-\frac{\partial U_{g}}{\partial y}\right) \frac{\partial \theta}{\partial y}\right]
\end{aligned}
$$

and

$$
\begin{aligned}
\mathbf{Q}_{y}=f_{o} \gamma[ & -\frac{\partial V_{g}}{\partial y} \frac{\partial \theta}{\partial y}-\frac{1}{2}\left(\frac{\partial V_{g}}{\partial x}+\frac{\partial U_{g}}{\partial y}\right) \frac{\partial \theta}{\partial x} \\
& \left.+\frac{1}{2}\left(\frac{\partial V_{g}}{\partial x}-\frac{\partial U_{g}}{\partial y}\right) \frac{\partial \theta}{\partial x}\right] .
\end{aligned}
$$

Now, we let $\zeta_{g}=\left(\partial V_{g} / \partial x-\partial U_{g} / \partial y\right)$ and $E_{2}=\left(\partial V_{g} / \partial x\right.$ $\left.+\partial U_{g} / \partial y\right)$ to represent the geostrophic vorticity and shearing deformation, respectively. With these substitutions, the component expressions for $\mathbf{Q}$ reduce to

$$
\mathbf{Q}_{x}=f_{o} \gamma\left(-\frac{\partial U_{g}}{\partial x} \frac{\partial \theta}{\partial x}-\frac{1}{2} E_{2} \frac{\partial \theta}{\partial y}-\frac{1}{2} \zeta_{g} \frac{\partial \theta}{\partial y}\right)
$$

and

$$
\mathbf{Q}_{y}=f_{o} \gamma\left(-\frac{\partial V_{g}}{\partial y} \frac{\partial \theta}{\partial y}-\frac{1}{2} E_{2} \frac{\partial \theta}{\partial x}+\frac{1}{2} \zeta_{g} \frac{\partial \theta}{\partial x}\right) .
$$

The stretching derivative in the first term of each expression in (B3) can be decomposed in a similar way into

$$
\frac{\partial U_{g}}{\partial x}=\frac{1}{2} \delta+\frac{1}{2} E_{1} \quad \text { and } \quad \frac{\partial V_{g}}{\partial y}=\frac{1}{2} \delta+\frac{1}{2} E_{1},
$$

where

$$
\delta=\left(\frac{\partial U_{g}}{\partial x}+\frac{\partial V_{g}}{\partial y}\right) \text { and } E_{1}=\left(\frac{\partial U_{g}}{\partial x}-\frac{\partial V_{g}}{\partial y}\right)
$$

represent the geostrophic divergence and stretching deformation, respectively.

Thus, the $\mathbf{Q}$ vector can be written in its component form as the sum of the geostrophic stretching and shearing deformations as well as the geostrophic vorticity as

$$
\mathbf{Q}_{x}=f_{o} \gamma\left(-\frac{1}{2} E_{1} \frac{\partial \theta}{\partial x}-\frac{1}{2} E_{2} \frac{\partial \theta}{\partial y}-\frac{1}{2} \zeta_{g} \frac{\partial \theta}{\partial y}\right)
$$

and

$$
\mathbf{Q}_{y}=f_{o} \gamma\left(\frac{1}{2} E_{1} \frac{\partial \theta}{\partial y}-\frac{1}{2} E_{2} \frac{\partial \theta}{\partial x}+\frac{1}{2} \zeta_{g} \frac{\partial \theta}{\partial x}\right) .
$$

Given the form of $\mathbf{Q}$ in (B4), it is easily shown that $\mathbf{Q}_{s}\left[\right.$ where $\left.\mathbf{Q}_{s}=(Q \cdot \hat{k} \times \boldsymbol{\nabla} \theta /|\boldsymbol{\nabla} \theta|)(\hat{k} \times \boldsymbol{\nabla} \theta /|\boldsymbol{\nabla} \theta|)\right]$ is given by

$$
\begin{aligned}
\mathbf{Q}_{s}=\frac{f_{o} \gamma}{|\boldsymbol{\nabla} \theta|^{2}} & \left\{\left[E_{1} \frac{\partial \theta}{\partial x} \frac{\partial \theta}{\partial y}+\frac{1}{2} E_{2}\left\{\left(\frac{\partial \theta}{\partial y}\right)^{2}-\left(\frac{\partial \theta}{\partial x}\right)^{2}\right\}\right]\right. \\
& \times(\hat{k} \times \boldsymbol{\nabla} \theta)\}+\frac{f_{o} \gamma}{2} \zeta_{g}(\hat{k} \times \boldsymbol{\nabla} \theta),
\end{aligned}
$$

which is identical to the form described in this paper with the first term in (B5) equal to $\mathbf{Q}_{\mathrm{DR}}$ and the second term equal to $\mathbf{Q}_{\mathrm{VR}}$. The form of $\mathbf{Q}$ in (B4) can also be used to show that the across-isentrope component of $\mathbf{Q}$ contains no contribution from geostrophic vorticity as $\mathbf{Q}_{n}=(Q \cdot \boldsymbol{\nabla} \theta /|\boldsymbol{\nabla} \theta|)(\boldsymbol{\nabla} \theta /|\boldsymbol{\nabla} \theta|)$ is given by

$\mathbf{Q}_{n}=\frac{f_{o} \gamma(\boldsymbol{\nabla} \theta)}{|\boldsymbol{\nabla} \theta|^{2}}\left[\frac{1}{2} E_{1}\left\{\left(\frac{\partial \theta}{\partial y}\right)^{2}-\left(\frac{\partial \theta}{\partial x}\right)^{2}\right\}-E_{2} \frac{\partial \theta}{\partial x} \frac{\partial \theta}{\partial y}\right]$.

\section{REFERENCES}

Barnes, S. L., and B. R. Colman, 1993: Quasigeostrophic diagnosis of cyclogenesis associated with a cutoff extratropical cycloneThe Christmas 1987 storm. Mon. Wea. Rev., 121, 1613-1634.

Davies, H. C., and A. M. Rossa, 1998: PV frontogenesis and upper tropospheric fronts. Mon. Wea. Rev., 126, 1528-1539.

Davies-Jones, R., 1991: The frontogenetical forcing of secondary circulations. Part I: The duality and generalization of the $\mathrm{Q}$ vector. J. Atmos. Sci., 48, 497-509.

Hakim, G., L. Bosart, and D. Keyser, 1996: The Ohio Valley wavemerger cyclogenesis event of 25-26 January 1978. Part II: Diagnosis using quasigeostrophic potential vorticity inversion. Mon. Wea. Rev., 124, 2176-2205.

Holton, J. R., 1992: An Introduction to Dynamical Meteorology. 3d ed. Academic Press, 511 pp.

Hoskins, B. J., and M. A. Pedder, 1980: The diagnosis of middle latitude synoptic development. Quart. J. Roy. Meteor. Soc., 106, 707-719.

- I. Draghici, and H. C. Davies, 1978: A new look at the $\omega$ equation. Quart. J. Roy. Meteor. Soc., 104, 31-38.

Keyser, D., M. J. Reeder, and R. J. Reed, 1988: A generalization of Petterssen's frontogenesis function and its relation to the forcing of vertical motion. Mon. Wea. Rev., 116, 762-780.

— B. D. Schmidt, and D. G. Duffy, 1992: Quasigeostrophic vertical motions diagnosed from along- and cross-isentrope components of the Q vector. Mon. Wea. Rev., 120, 731-741.

Kurz, M., 1997: The role of frontogenetic and frontolytic wind field effects during cyclone development. Meteor. Appl., 4, 353-363.

Martin, J. E., 1998a: On the deformation term in the quasigeostrophic omega equation. Mon. Wea. Rev., 126, 2000-2007.

_ 1998b: The structure and evolution of a continental winter cyclone. Part I: Frontal structure and the classical occlusion process. Mon. Wea. Rev., 126, 303-328.

- 1999: Quasigeostrophic forcing of ascent in the occluded sector of cyclones and the trowal airstream. Mon. Wea. Rev., 127, 7088.

McIntyre, M. E., and T. N. Palmer, 1983: Breaking planetary waves in the stratosphere. Nature, 305, 593-600.

Petterssen, S., 1936: A contribution to the theory of frontogenesis. Geofys. Publ., 11, 1-27.

— storms. Quart. J. Roy. Meteor. Soc., 97, 457-482.

Sutcliffe, R. C., 1947: A contribution to the problem of development. Quart. J. Roy. Meteor. Soc., 73, 370-383.

Trenberth, K. E., 1978: On the interpretation of the diagnostic quasigeostrophic omega equation. Mon. Wea. Rev., 106, 131-137.

Wiin-Nielsen, A., 1959: On a graphical method for an approximate determination of the vertical velocity in the mid-troposphere. Tellus, 11, 432-440. 